\title{
A NOTE ON TRANSLATION INVARIANTS
}

ROY L. ADLER AND ALAN G. KONHEIM

1. Introduction. Autocorrelation functions play a central role in many engineering applications. The following operation describes a certain optical system [2]: from a function $f$ of two variables, representing the transparency of a photographic slide, another transparency function $F$ is produced on a photographic plate which is the autocorrleation function of $f$; that is,

$$
F(x, y)=\int_{-\infty}^{\infty} \int_{-\infty}^{\infty} f(\xi, \eta) f(\xi-x, \eta-y) d \xi d \eta .
$$

The essential feature of the function $F$ is that it remains unchanged if $f$ is replaced by $f^{*}$ where $f^{*}(\xi, \eta)=f(\xi+\alpha, \eta+\beta)$. A uniqueness question now arises; if $f$ and $g$ have the same autocorrelation function, are they related by a rectilinear motion of the plane?

The answer to this question is negative as is shown in $\S 4$ by counterexample. In $\S 2$ we present this problem in a more general setting and in $\S 3$ the main theorem.

2. Notation and definitions. Let $G$ be a locally compact abelian group, $\mu$ Haar measure, and $L_{1, r}(G)$ the space of real-valued $\mu$-integrable functions on $G$. For each $s \in G$ define the translation operator $T_{s}: L_{1, r}(G) \rightarrow L_{1, r}(G)$ by $\left(T_{s} f\right)(x)=f(s x)$ and denote by $\mathfrak{I}(f)$ the set $\left\{g: g \in L_{1, r}(G),\left(T_{s} f\right)(x)=g(x)(\mu\right.$ a.e. $)$ for some $\left.s \in G\right\}$ of translates of $f$. A functional $\rho(\cdot)$ with domain $L_{1, r}(G)$ will be called translation invariant if $\rho(f)=\rho(g)$ for all $g \in \mathfrak{T}(f)$. A class of translation invariants $\mathfrak{B}=\left\{\rho_{\omega}(\cdot): \omega \in \Omega\right\}$ is complete if $\rho_{\omega}(f)=\rho_{\omega}(g)$ for all $\omega \in \Omega$ implies $g \in \mathfrak{T}(f)$.

The kth order autocorrelation function of $f \in L_{1, r}(G)$ is the realvalued function $\rho_{k}(f) \equiv \rho_{k}(f)\left(x_{1}, x_{2}, \cdots, x_{k}\right)$ with domain $G^{(k)}$ $=G \times G \times \cdots \times G$ ( $k$ copies $)$ defined formally by

$$
\rho_{k}(f)\left(x_{1}, x_{2}, \cdots, x_{k}\right)=\int_{G} f(\xi) f\left(\xi x_{1}\right) f\left(\xi x_{2}\right) \cdots f\left(\xi x_{k}\right) \mu(d \xi) .
$$

The proof that $\rho_{k}(f)$ is $\mu^{(k)}$-almost everywhere ${ }^{1}$ defined and in $L_{1, r}(G)$ is a modification of the argument of $[1$, p. 121] used in establishing that the convolution of integrable functions is integrable. The map-

Presented to the Society, January 23, 1961; received by the editors December 10, 1960 and, in revised form, March 20, 1961.

${ }_{1}^{1} \mu^{(k)}$ denotes the direct product measure on $G^{(k)}$ given by $\mu$ on $G$. 
ping $f \rightarrow \rho_{k}(f)$ of $L_{1, r}(G)$ into $L_{1, r}\left(G^{(k)}\right)$ is a translation invariant functional as $\mu$ is Haar measure.

Let $\hat{G}$ denote the character group of $G$. For $h \in L_{1, r}\left(G^{(k)}\right)$ define the " $k$-dimensional" Fourier transform by

$$
\hat{h}\left(\chi_{1}, \cdots, \chi_{k}\right)=\int_{G^{(k)}} \prod_{i=1}^{k} \overline{\chi_{i}}\left(\xi_{i}\right) h\left(\xi_{1}, \cdots, \xi_{k}\right) \mu^{(k)}\left(d \xi_{1} \times \cdots \times d \xi_{k}\right)
$$

where $\chi_{i} \in \hat{G}$ and - denotes complex conjugation. For $f \in L_{1, r}(G)$ let $\sigma(f)=\{\chi: \chi \in \hat{G}, \hat{f}(\chi) \neq 0\}$.

If $K$ is a group and $H$ a subset of $K$, we shall say that a mapping $\phi, \phi: H \rightarrow C=\{\exp (2 \pi i x): 0 \leqq x<1\}$ acts homomorphically on $H$ to $C$ if

(i) $\phi\left(a^{-1}\right)=[\phi(a)]^{-1}$ whenever $a, a^{-1} \in H$.

(ii) For each $N, \phi\left(\prod_{i=1}^{N} a_{i}\right)=\prod_{i=1}^{N} \phi\left(a_{i}\right)$ whenever $a_{1}, a_{2}, \cdots, a_{N}$ and $\prod_{i=1}^{N} a_{i}$ are in $H$.

\section{Main theorem.}

TheOREM. $\left\{\rho_{k}(\cdot): k=1,2, \cdots\right\}$ is a complete set of translation invariants for $L_{1, r}(G)$.

LEMma 1. Let $H$ be a symmetric subset of a group $K$ (i.e., if $a \in H$ then $\left.a^{-1} \in H\right)$. If $\phi$ acts homomorphically on $H$ to $C$ then $\phi$ can be extended to a homomorphism of the subgroup $[H]$, generated by $H$, to $C$.

Proof. For $c \in[H]$ define $\phi(c)=\phi\left(a_{1}\right) \phi\left(a_{2}\right) \cdots \phi\left(a_{p}\right)$ where $c=a_{1} a_{2} \cdots a_{p}, a_{i} \in H(1 \leqq i \leqq p)$. To see that this definition of $\phi(c)$ is consistent let us suppose $c=b_{1} b_{2} \cdots b_{q}$ with $b_{i} \in H(1 \leqq i \leqq q)$. Then $b_{q}=a_{1} a_{2} \cdots a_{p} b_{1}^{-1} b_{2}^{-1} \cdots b_{q-1}^{-1}$ so that $\phi\left(b_{q}\right)=\phi\left(a_{1}\right) \phi\left(a_{2}\right) \cdots$ $\phi\left(a_{p}\right) \phi\left(b_{1}^{-1}\right) \phi\left(b_{2}^{-1}\right) \cdots \phi\left(b_{q-1}^{-1}\right)$ by (ii) above. By (i) $\phi\left(b_{i}^{-1}\right)=\left[\phi\left(b_{i}\right)\right]^{-1}$ so that $\phi\left(b_{1}\right) \phi\left(b_{2}\right) \cdots \phi\left(b_{q}\right)=\phi\left(a_{1}\right) \phi\left(a_{2}\right) \cdots \phi\left(a_{p}\right)$. The extended mapping is easily seen to be a homomorphism of $[H]$ into $C$.

LeMma 2. If $K$ is a topological group, $H$ an open symmetric subset of $K$ and $\phi$ a continuous mapping $\phi: H \rightarrow C$ which acts homomorphically on $H$ to $C$, then $\phi$ can be extended to a continuous homomorphism of the (necessarily closed) subgroup $[H]$, generated by $H$, to $C$.

Proof. By Lemma 1, $\phi$ can be extended to $\phi^{*}$ an algebraic homomorphism of $[H]$ to $C$. It suffices to verify that $\phi^{*}$ is continuous. Let $H_{n}$ denote the set $H \cdot H \cdot \cdots \cdot H$ ( $n$ copies) $;^{2} H_{n}$ is clearly an open subset of $K$. Let $\phi_{n}$ denote the restriction of $\phi^{*}$ to $H_{n}$. Clearly $\phi_{n}$ is continuous on $H_{n}$ and since $[H]=\bigcup_{n=1}^{\infty} H_{n}, \phi^{*}$ is continuous on [H]. As $[H]$ is an open subgroup of $K$ it follows that it is necessarily a closed subgroup [3, p. 37].

\footnotetext{
${ }^{2} H_{n}=\left\{c: c=a_{1} a_{2} \cdots a_{n}, a_{i} \in H\right\}$.
} 
Proof of the theorem. If $f \in L_{1, r}(G)$ then

$$
\hat{f}\left(\chi^{-1}\right)=\bar{f}(\chi)
$$

and hence $\sigma(f)$ is a symmetric subset of $\hat{G}$. An elementary computation using Fubini's theorem yields

$$
\hat{\rho}_{k}(f)\left(\chi_{1}, \chi_{2}, \cdots, \chi_{k}\right)=\hat{f}\left(\prod_{i=1}^{k} \chi_{i}^{-1}\right) \prod_{i=1}^{k} \hat{f}\left(\chi_{i}\right)
$$

Suppose $f, g \in L_{1, r}(G)$ with

$$
\rho_{k}(f)=\rho_{k}(g), \quad k=1,2, \cdots
$$

From (2) with $k=1$ we obtain

$$
\hat{f}(\chi) \hat{f}\left(\chi^{-1}\right)=|\hat{f}(\chi)|^{2}=|\hat{g}(\chi)|^{2}=\hat{g}(\chi) \hat{g}\left(\chi^{-1}\right)
$$

so that we may write

$$
\hat{g}(\chi)=\phi(\chi) f(\chi)
$$

where $|\phi(\chi)|=1$ for $\chi \in \sigma(f)$. The reality of $f$ and $g$ and (4) implies moreover

$$
\phi\left(\chi^{-1}\right)=\overline{\phi(\chi)}=[\phi(\chi)]^{-1}
$$

again for $\chi \in \sigma(f)$. Equations (1), (2), (4) and (5) yield

whenever

$$
\phi\left(\prod_{i=1}^{N} \chi_{i}\right)=\prod_{i=1}^{N} \phi\left(\chi_{i}\right)
$$

$$
\chi_{1}, \chi_{2}, \cdots, \chi_{N}, \prod_{i=1}^{N} \chi_{i} \in \sigma(f) .
$$

Since $\hat{f}(\cdot)$ is continuous and does not vanish on $\sigma(f), \phi(\cdot)$ is continuous on $\sigma(f)$ and $\sigma(f)$ is open. Thus $\phi$ is continuous and acts homomorphically on the open symmetric subset $\sigma(f)$ to $C$. By Lemma 2, $\phi$ can be extended to a continuous homomorphism of $[\sigma(f)]$ to $C$. The mapping $\phi:[\sigma(f)] \rightarrow C$ can be extended $[4$, p. 138] to a character of $\hat{G}$. Thus we have

$$
\hat{g}(\chi)=\phi(\chi) \hat{f}(\chi)
$$

for all $\chi \in \hat{G}$, with $\phi$ a character of $\hat{G}$. By the duality theorem [1, p. 151]

$$
\phi(\chi)=\chi(a)
$$

for some fixed $a \in G$ and therefore the uniqueness theorem $[1$, p. 146] finally yields

$$
g(x)=f(a x) \quad(\mu \text { a.e. })
$$


4. Counterexamples. We will now exhibit a counterexample to the conjecture

$$
\rho_{1}(f)=\rho_{1}(g) \Rightarrow g \in \mathfrak{T}(f) .
$$

Let $G$ be the real line and $A$ a subset of $G$ of finite positive measure which is not a translate of its inverse set. If $f$ and $g$ are the characteristic functions of $A$ and $-A=\{-x: x \in A\}$ respectively then $\rho_{1}(f)(x)=\mu(A \cap(A-x))=\mu(-A \cap(-A-x))=\rho_{1}(g)(x)$ while $g \notin \mathfrak{T}(f)$. Similarly the conjecture

$$
\rho_{1}(f)=\rho_{1}(g) \Rightarrow g \in \mathfrak{T}(f) \cap \mathfrak{I}\left(f^{-}\right)
$$

where $f^{-}(x)=f\left(x^{-1}\right)$ is also false. To show this let $A$ and $B$ be subsets of the real line as described above and $f$ and $g$ the characteristic functions of $A \times B$ and $A \times(-B)$. Then $f$ and $g$ have the same (first order) autocorrelation function while $g \in \mathfrak{T}(f) \cup \mathfrak{T}\left(f^{-}\right)$.

It is likewise conjectured by the authors that no finite subset of $\left\{\rho_{k}(\cdot): k=1,2, \cdots\right\}$ is a complete set of translation invariants unless restrictions are placed upon the functions. In this connection the following corollary is easily obtained.

Corollary. If $\mathfrak{U}_{,}$is the subset of $L_{1, r}(G)$ consisting of those $f$ for which $\sigma(f) \cdot \sigma(f) \cdot \cdots \cdot \sigma(f)$ ( $\nu$ copies) is a subgroup of $\hat{G}$ then $\left\{\rho_{k}(\cdot): k=1,2, \cdots, 3 \nu-1\right\}$ is a complete set of translation invariants for $\mathfrak{U}_{.}$. In particular if $\hat{f}(\chi) \neq 0(\chi \in \hat{G})$ and $\rho_{i}(f)=\rho_{i}(g)(i=1,2)$ then $g \in \mathfrak{T}(f)$.

5. Further considerations. For complex-valued $\mu$-integrable functions $\sigma(f)$ is no longer symmetric in general and our argument fails. In fact if $G$ is the unit interval and $f \in L_{1}(G)$ with

$$
\int_{0}^{1} f(\xi) \exp (-2 \pi i n \xi) d \xi=0\left\{\begin{array}{l}
n<0, \\
n>N
\end{array}\right.
$$

then $\rho_{k}(f) \equiv 0$ for all $k$. Minor modifications of the definition of the autocorrelation function do not substantially change the situation.

\section{REFERENCES}

1. L. H. Loomis, An introduction to abstract harmonic analysis, Van Nostrand, New York, 1953.

2. W. Meyer-Eppler and G. Darius, Two-dimensional photographic autocorrelation of pictures and alphabet letters, Proc. 3rd London Sympos. Information Theory, pp. 34-36.

3. D. Montgomery and L. Zippen, Topological transformation groups, Interscience, New York, 1955.

4. L. Pontrjagin, Topological groups, Princeton Univ. Press, Princeton, N. J., 1946.

ibM Research Center, Yorktown Heights, New York 